\title{
Model for monitoring of a charge qubit using a radio-frequency quantum point contact including experimental imperfections
}

\author{
Neil P. Oxtoby, 1,2 , J Jay Gambetta, ${ }^{3,4}$ and H. M. Wiseman ${ }^{1, \text { 田 }}$ \\ ${ }^{1}$ Centre for Quantum Computer Technology, Centre for Quantum Dynamics, \\ School of Biomolecular and Physical Sciences, Griffith University, Brisbane 4111, Australia \\ ${ }^{2}$ Quantum Physics Group, STRI, School of Physics, Astronomy and Mathematics, \\ University of Hertfordshire, Hatfield, Herts AL10 9AB, United Kingdom \\ ${ }^{3}$ Institute for Quantum Computing and Department of Physics and Astronomy, \\ University of Waterloo, Waterloo, Ontario N2L 3G1, Canada \\ ${ }^{4}$ Department of Applied Physics and Department of Physics, \\ Yale University, New Haven, Connecticut 06520, USA
}

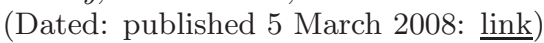

\begin{abstract}
The extension of quantum trajectory theory to incorporate realistic imperfections in the measurement of solid-state qubits is important for quantum computation, particularly for the purposes of state preparation and error-correction as well as for readout of computations. Previously this has been achieved for low-frequency (dc) weak measurements. In this paper we extend realistic quantum trajectory theory to include radio frequency ( $\mathrm{rf}$ ) weak measurements where a low-transparency quantum point contact (QPC), coupled to a charge qubit, is used to damp a classical oscillator circuit. The resulting realistic quantum trajectory equation must be solved numerically. We present an analytical result for the limit of large dissipation within the oscillator (relative to the QPC), where the oscillator slaves to the qubit. The $\mathrm{rf}+\mathrm{dc}$ mode of operation is considered. Here the QPC is biased (dc) as well as subjected to a small-amplitude sinusoidal carrier signal (rf). The $\mathrm{rf}+\mathrm{dc}$ $\mathrm{QPC}$ is shown to be a low-efficiency charge qubit detector, that may nevertheless be higher than the dc-QPC (which is subject to $1 / f$ noise).
\end{abstract}

PACS numbers: 73.23.Hk, 03.67.Lx

\section{INTRODUCTION}

Solid-state proposals for building scalable quantum information processors $1,2,3,4,5$ seem promising. In any quantum information processor, the quantum bits (qubits) of information need to be read out, as well as controlled (via measurement-based feedback, for example). Quantum trajectory theory $\underline{6,7,8}$ has been used to describe single realizations of the continuous-intime weak quantum measurement of electronic charge qubits $9,10,11,12,13,14,15,16,17,18,19$ conditioned by the electrical output of a mesoscopic measurement device such as a quantum point contact (QPC) or single-electron transistor (SET). Very recently, quantum trajectory theory has been applied to circuit QED $\underline{20}$ In these works the qubit evolution was conditioned on idealized measurement results (such as electron tunnelling) at the scale of the mesoscopic detector. That is, the observer's state of knowledge about the qubit state is updated based on these idealized measurement results. Two of us recently extended such work to condition the qubit state on a macroscopic signal that is realistically available to an observer in a dc-QPC measurement $\stackrel{21}{=}$ In particular, this extension considered the noisy, filtering characteristic of an external circuit, including an amplifier. The result is a corrupted version of the idealized measurement results upon which the qubit evolution can be conditioned. This extension is known as "realistic quantum trajectory" theory and was pioneered for photodetection in quantum optics, $\stackrel{22,23}{=}$ where it was applied to an avalanche photo- diode and a photoreceiver.

In traditional dc charge-qubit measurement techniques, low frequency noise (" $1 / f$ noise" 24,25$)$ limits the detector sensitivity $\underline{26}$ To circumvent this, Schoelkopf et $a l \underline{\underline{26}}$ introduced the so-called radio-frequency singleelectron transistor (rf-SET). For the original configuration, the rf-SET demonstrated constant gain from dc to $100 \mathrm{MHz}$ - an improvement on the conventional SET bandwidth by two orders of magnitude $\underline{26}$ The idea is to measure the damping of a resonant (oscillator) circuit in which the SET is embedded. In the context of charge-qubit detection, 27 the damping depends on the qubit state, via the SET. Thus, monitoring the damping of the oscillator constitutes a continuous-in-time measurement of the charge qubit. This concept can be applied to any charge-sensitive detector, in particular to the QPC, for example ${ }^{28}$

In this paper we derive an evolution equation for the conditional state of a charge qubit monitored continuously in time by a detector operated in the rf configuration. To the best of our knowledge, an equation of this type has not previously been derived. We also consider conditioning the qubit state on measurement results available to a realistic observer, within the framework of realistic quantum trajectory theory $\underline{21,22,23,29}$ In this approach, the bare charge-qubit detector (QPC in our case) is embedded in a realistic circuit, and an equation is derived that describes the evolution of the combined circuit-plus-qubit state conditioned on measurement results available to a realistic observer. 
Being able to determine the state of a quantum system conditioned on actual measurement results is expected to be vitally important for quantum computation, particularly for state preparation and quantum error correction, $30,31,32,33$ More broadly, it is also essential for understanding and designing optimal feedback control $10,34,35,36,37,38,39,40,41,42,43$

To simplify our analysis, we make a number of approximations. First, we use the low-transparency QPC as the charge-sensitive detector embedded in the rf circuit, instead of the SET. Second, we make a rotating wave approximation (RWA) to remove the $1 / f$ noise from our equations (as the rf configuration removes it in the experiment). In order to do this, we assume the QPC to be operating in the weakly-responding (diffusive) limit, $\underline{9}, 10,12$ In this limit, the QPC shot noise appears as white noise, which is modeled as a Gaussian-distributed stochastic process (having a diffusive appearance). Third, we assume that the rf-QPC is operated in the $\mathrm{rf}+\mathrm{dc}$ mode introduced in the context of a SET in Ref. 44. This is where the QPC is subjected to a small amplitude sinusoidal oscillation (rf) superposed on a relatively large bias (dc). We find that the $\mathrm{rf}+\mathrm{dc}$ QPC is a highly inefficient charge-qubit detector. However, this may be higher than the measurement efficiency of the dc-QPC, which in practice is not only limited by $1 / f$ noise, but is also further degraded the longer the measurement has to continue.

The paper is organized as follows. The qubit and QPC are discussed in Sec. III The classical circuit is discussed and analyzed in Sec. III including the presentation of stochastic differential equations describing the state of the oscillator circuit. The stochastic master equation for the qubit state conditioned on the bare detector output is presented in Sec. IV] The realistic quantum trajectory derivation then proceeds in an analogous manner to Ref. 21, where the circuit state was described by one parameter - the charge on a capacitor. In general the oscillator circuit needs two parameters to describe its state, thus hinting at a complication in deriving the realistic equations for the rf configuration. However, because the QPC only damps the oscillator circuit (and doesn't induce a phase-shift in the reflected signal), all of the qubit information is contained in the amplitude (damping) of the reflected signal. Therefore we can proceed with the realistic quantum trajectory derivation using a single parameter to describe the state of the classical circuit. As always, numerical calculations must be performed to obtain the realistic quantum trajectories. However, in Sec. VI we present analytical results for the heavily damped limit where the circuit is slaved to the qubit (adiabatic elimination of the circuit). The paper is concluded in Sec. VII.

\section{QUBIT AND QPC}

The measured quantum system we consider is the double-quantum-dot (DQD) charge qubit. $\stackrel{45,46}{ } \mathrm{~A}$ schematic of the isolated $\mathrm{DQD}^{46}$ and capacitively coupled QPC is shown in Fig. 11. We consider the lowtransparency QPC, and represent it as a tunnel barrier between source and drain leads (reservoirs) with respective Fermi levels $\mu_{\mathrm{S}}$ and $\mu_{\mathrm{D}}$. The QPC voltage bias is $e V_{\mathrm{d}}=\mu_{\mathrm{S}}-\mu_{\mathrm{D}}$, where $e>0$ is the quantum of electronic charge ${ }^{62}$ The DQDs are occupied by a single excess electron, the location of which determines the charge state of the qubit. The charge basis states are denoted $|0\rangle$ and $|1\rangle$ (see Fig. 11). We assume that each quantum dot has only one single-electron energy level available for occupation by the qubit electron, denoted by $E_{1}$ and $E_{0}$ for the near and far dot, respectively.

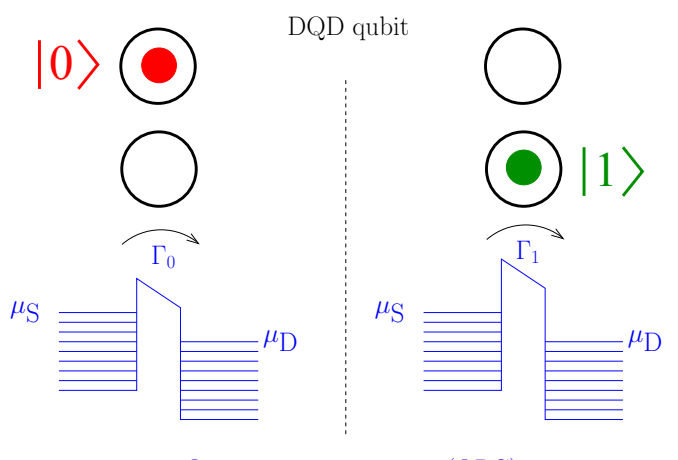

Quantum point contact (QPC)

FIG. 1: Schematic of an isolated DQD qubit and capacitively coupled low-transparency QPC between source $(\mathrm{S})$ and drain (D) leads.

The Hamiltonian for the qubit can be written as

$$
\hat{H}_{\mathrm{qb}}=\frac{1}{2}\left(\varepsilon \hat{\sigma}_{z}+\Omega_{\mathrm{tun}} \hat{\sigma}_{x}\right),
$$

where the qubit energy asymmetry is $\varepsilon \equiv E_{1}-E_{0}, \Omega_{\text {tun }}$ is the DQD tunnel-coupling strength, and $\hat{\sigma}_{x, z}$ are Pauli matrices in the measurement (charge) basis. The eigenvalues of $\hat{H}_{\mathrm{qb}}$ are $\mp \Omega / 2$, where $\Omega \equiv \sqrt{\Omega_{\text {tun }}^{2}+\varepsilon^{2}}$.

Associated with each of the qubit charge states is a current through the detector. The average current through the detector is $I_{1}=e \Gamma_{1}$ when the nearby dot is occupied, and $I_{0}=e \Gamma_{0}$ when the nearby dot is unoccupied. The variation in the detector output that depends on the qubit state is referred to as the detector's response and is denoted $\Delta I \equiv I_{1}-I_{0}$. We can quantify the strength of the detector response by $0 \leq|\Delta I| / I_{\mathrm{av}} \leq 2$, where $I_{\mathrm{av}} \equiv\left(I_{1}+I_{0}\right) / 2$. Thus, a weakly responding ${ }^{9}$ detector satisfies $|\Delta I| \ll I_{\mathrm{av}}$, and a detector with finite, or strong, response satisfies $|\Delta I| \sim I_{\mathrm{av}}$. In this paper we consider the limit of a weakly responding $\mathrm{QPC}$, where the $\mathrm{QPC}$ shot noise appears as a diffusive, white noise process.

The total Hamiltonian for the system is

$$
\hat{H}_{\mathrm{Tot}}=\hat{H}_{\mathrm{qb}}+\hat{H}_{\mathrm{F}}+\hat{H}_{\mathrm{T}}+\hat{H}_{\text {coup }},
$$

where the qubit Hamiltonian $\hat{H}_{\mathrm{qb}}$ is given by Eq. (1). The free Hamiltonian describing the continua of electron 
channels (momenta) $k$ and $q$ in the source and drain leads is

$$
\hat{H}_{\mathrm{F}}=\sum_{k} \omega_{\mathrm{S} k} \hat{a}_{\mathrm{S} k}^{\dagger} \hat{a}_{\mathrm{S} k}+\sum_{q} \omega_{\mathrm{D} q} \hat{a}_{\mathrm{D} q}^{\dagger} \hat{a}_{\mathrm{D} q}
$$

where $\hat{a}_{\mathrm{S}}$ and $\hat{a}_{\mathrm{D}}$ are the Fermi field annihilation operators for the source and drain leads, respectively. The tunnelling Hamiltonian

$$
\hat{H}_{\mathrm{T}}=\sum_{k, q} T_{k q} \hat{a}_{\mathrm{S} k}^{\dagger} \hat{a}_{\mathrm{D} q}+T_{q k}^{*} \hat{a}_{\mathrm{D} q}^{\dagger} \hat{a}_{\mathrm{S} k}
$$

describes tunnelling between the source and drain leads. The probability amplitude for a source electron in channel $k$ to tunnel through the QPC into the drain channel $q$ is $T_{k q}$. The coupling Hamiltonian

$$
\hat{H}_{\text {coup }}=\hat{n}\left(\sum_{k, q} \chi_{k q} \hat{a}_{\mathrm{S} k}^{\dagger} \hat{a}_{\mathrm{D} q}+\chi_{q k}^{*} \hat{a}_{\mathrm{D} q}^{\dagger} \hat{a}_{\mathrm{S} k}\right)
$$

describes the change in the effective QPC tunnelling amplitude from $T_{k q} \rightarrow T_{k q}+\chi_{k q}$ when the nearby dot is occupied. This changes the QPC current from $I_{0}=e|\mathcal{T}|^{2}$ to $I_{1}=e|\mathcal{T}+\mathcal{X}|^{2}$. Here $\mathcal{T} \propto T_{k q}$ and $\mathcal{X} \propto \chi_{k q}$ are both proportional to the square-root of the source-drain voltage $V_{\mathrm{d}}, \underline{12}, \underline{\underline{6}}$ The occupation number operator of the nearby dot is $\hat{n}=\left(1+\hat{\sigma}_{z}\right) / 2$. Note that the height of the QPC (tunnel-junction) barrier is increased when the nearby dot is occupied, due to electrostatic repulsion, so that $I_{0}>I_{1}$.

\section{CLASSICAL SYSTEM: OSCILLATOR}

An oscillator circuit, or tank circuit, consists of an inductor $L$, and capacitor $C$. We treat the oscillator classically. The (angular) frequency for which resonance occurs in such an unloaded tank circuit, $\omega_{0}=1 / \sqrt{L C}$, is known as the resonance frequency. Embedding a dissipative component, like a resistor, into the tank circuit provides a source of damping. For our purposes, the QPC provides the damping, so the oscillator damping depends on the qubit state via the qubit-dependent QPC conductance. It therefore makes sense to monitor the damping of the tank circuit in order to ascertain the qubit state. This is achieved by using the tank circuit to terminate a transmission line. Impedance mismatch between the tank circuit and transmission line causes a signal launched towards the tank circuit to be reflected back along the transmission line, where the reflected signal can be observed $\underline{64}$ See Fig. 2 for our equivalent circuit representing the rf-QPC setup. A recent experiment ${ }^{28}$ reports realization of a semiconductor $\mathrm{rf}-$ PC (radio-frequency point contact), with two benefits over the SET - lower PC impedance (thus simplifying impedance matching with the transmission line), and easier fabrication. This as yet unoptimized device exhibits a lower charge sensitivity than the rf-SET. In said experiment, the point contact is operating as a simple voltage-controlled resistor rather than a QPC. Another more recent experiment $\frac{47}{}$ has realized fast charge sensing with a semiconductor rf-QPC.

The AC voltage signal launched towards the tank circuit is referred to as the "carrier" 26 Using a carrier signal frequency equal to the resonance frequency of the unloaded tank circuit allows the detector to be replaced by its (frequency-dependent) dynamic resistance $R_{\mathrm{d}} \underline{\underline{44}, \underline{48}}$ This is a valid first-order approximation since the tank circuit is most sensitive to frequencies within a small bandwidth around the resonance frequency.

Consider the equivalent circuit of Fig. 2. The oscillator circuit consisting of an inductance $L$ and capacitance $C$ terminates the transmission line of impedance $Z_{\mathrm{TL}}=$ $50 \Omega$. The voltages (potential drops) across the oscillator components can be written as

$$
\begin{aligned}
V_{\mathrm{L}}(t) & =\dot{\Phi}(t), \\
V_{\mathrm{C}}(t) & =\frac{Q(t)}{C},
\end{aligned}
$$

where $\Phi(t)$ is the flux through the inductor, and $Q(t)$ is the charge on the capacitor. These variables represent conjugate variables that together completely characterize the classical oscillator state. The voltage across the detector, $V_{\mathrm{d}}(t)$, is the topic of the following subsection. The current flowing through the detector, $I(t)$, will be discussed in Sec. IV] We define the incoming (relative to the tank circuit) transmission line voltage as the carrier signal $V_{\mathrm{c}}(t)=V_{\text {in }}^{\mathrm{rf}} \cos \left(\omega_{0} t\right)$ plus some noise $N(t)$ :

$$
V_{\text {in }}(t)=V_{\mathrm{c}}(t)+N(t) .
$$

The outgoing voltage in the transmission line is denoted $V_{\text {out }}(t)$. As in the experiments of Ref. 49, the detector bias $V_{0}$ is added at a bias tee at the end of the transmission line.

\section{A. Detector voltage}

In Sec. IV we will discuss the conditional dynamics of the quantum system undergoing rf-QPC monitoring. Since the QPC tunnelling rates depend on the detector voltage $V_{\mathrm{d}}(t)$ (see Ref. 12), we calculate it here.

Using complex phasor notation, the voltage divider rule gives the detector voltage as

$$
V_{\mathrm{d}}(t)=V_{0}+\operatorname{Re}\left[\frac{Z_{\mathrm{RC}}}{Z} V(t)\right]
$$

since the inductor is a "short-circuit" at dc. The impedances here are $Z_{\mathrm{RC}}^{-1}=R_{\mathrm{d}}^{-1}+i \omega_{0} C\left(R_{\mathrm{d}} C\right.$ combination), and $Z=Z_{\mathrm{RC}}+Z_{\mathrm{L}}$ (entire tank circuit), with $Z_{\mathrm{L}}=i \omega_{0} L$ (inductor). Here the AC voltage in the transmission line,

$$
V(t)=(1+\beta) V_{\mathrm{c}}(t)
$$




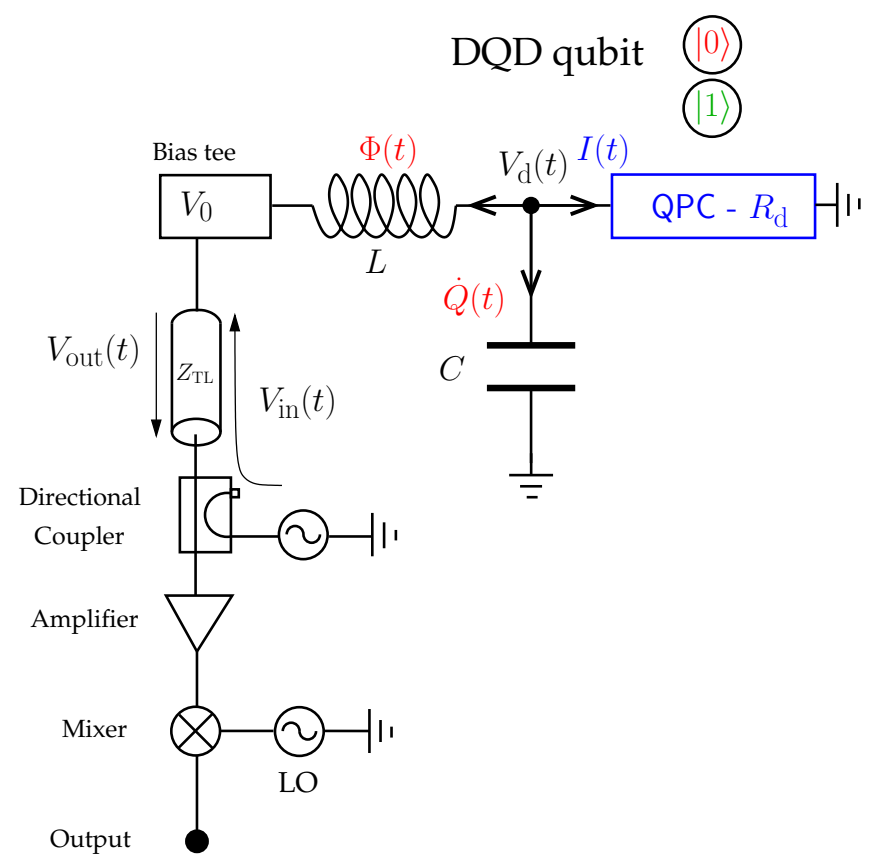

FIG. 2: Equivalent circuit for continuous monitoring of a charge qubit coupled to a classical $L C$ oscillator with inductance $L$ and capacitance $C$. We consider the charge-sensitive detector that loads the oscillator circuit to be a QPC (see Fig. 1 for details). Measurement is achieved using reflection with the input voltage, $V_{\text {in }}(t)$, and the output voltage, $V_{\text {out }}(t)$, being separated by a directional coupler. The output voltage is then amplified and mixed with a local oscillator, $L O$, and then measured.

consists of the incident and reflected AC signal. Here we make an assumption that $N(t)$ comprises predominantly low-frequency noise which will be removed by the rf-QPC, and so we drop it to simplify the analysis. The reflection coefficient, $\beta$, is given in terms of the impedance mismatch between tank circuit and transmission line as

$$
\beta=\frac{Z-Z_{\mathrm{TL}}}{Z+Z_{\mathrm{TL}}}
$$

That is, the AC signal reflected off the loaded tank circuit is given by $\beta V_{\mathrm{c}}(t)$. Combining these results, the detector voltage can be written as

$$
\begin{aligned}
V_{\mathrm{d}}(t) & =V_{0}+2 \operatorname{Re}\left[\frac{Z_{\mathrm{RC}}}{Z+Z_{\mathrm{TL}}} V_{\mathrm{c}}(t)\right] \\
& =V_{0}+2 \operatorname{Re}\left[G e^{i \phi} V_{\mathrm{c}}(t)\right] .
\end{aligned}
$$

Here the amplitude gain $G$ and phase-shift $\phi$ of the carrier signal when it reaches the QPC are given in terms of the circuit and detector quality factors as

$$
\begin{aligned}
G & \equiv\left(Q_{\mathrm{d}}^{-2} Q^{-2}+Q_{\mathrm{T}}^{-2}\right)^{-1 / 2}, \\
\tan (\phi) & =Q_{\mathrm{T}} .
\end{aligned}
$$

The quality factors of the unloaded tank circuit, detector, and loaded tank circuit are $Q \equiv \omega_{0} / \gamma, Q_{\mathrm{d}} \equiv \gamma_{\mathrm{d}} / \omega_{0}$, and $Q_{\mathrm{T}}^{-1} \equiv Q^{-1}+Q_{\mathrm{d}}^{-1}$, respectively.

In the high quality limit $Q_{\mathrm{d}}, Q \gg 1\left(\gamma_{\mathrm{d}} \gg \omega_{0} \gg \gamma\right)$, the tank circuit damping is due primarily to the detector. This limit therefore represents the highest sensitivity for the $\mathrm{rf}+\mathrm{dc}$ QPC. We find that $G \sim Q_{\mathrm{T}}$ and $\phi \sim \pi / 2$, so that the voltage across the QPC experiences a negative $\pi / 2$ phase-shift ( $\cos \rightarrow \sin )$ after the inductor. This is expected since the voltage across an (ideal) inductor lags the driving signal by $\pi / 2$. In this limit the voltage across the QPC is

$$
V_{\mathrm{d}}(t)=V_{0}\left[1+\epsilon_{\mathrm{in}} \sin \left(\omega_{0} t\right)\right] .
$$

Here we have defined the dimensionless parameter $\epsilon_{\text {in }} \equiv$ $2 Q_{\mathrm{T}} V_{\mathrm{in}}^{\mathrm{rf}} / V_{0}$, which satisfies $\epsilon_{\text {in }} \ll 1$ in the $\mathrm{rf}+\mathrm{dc}$ mode $\underline{\underline{44}}$ Equation (13) is in agreement with Ref. 48.

\section{B. Idealized classical dynamics}

The two conjugate parameters we use to describe the oscillator state are the flux through the inductor, $\Phi(t)$, and the charge on the capacitor, $Q(t)$. The dynamics of the oscillator are found by analyzing the equivalent circuit of Fig. 2 using the well-known Kirchhoff circuit laws. Doing this we find that the classical system obeys the following set of coupled differential equations

$$
\begin{aligned}
\dot{\Phi}(t) & =-\gamma \Phi(t)+\frac{Q(t)}{C}-V_{0}-2\left[V_{\mathrm{c}}(t)+N(t)\right] \\
\dot{Q}(t) & =-\frac{\Phi(t)}{L}-I(t), \\
V_{\text {out }}(t) & =V_{\mathrm{c}}(t)+N(t)+\frac{Z_{\mathrm{TL}} \Phi(t)}{L}
\end{aligned}
$$

where $I(t)$ is detector current and we have re-included the input noise $N(t)$. These are the equations of a damped harmonic oscillator driven by both the input voltage and the QPC current (including the shot noise) at frequency $\omega_{0}$. The $1 / f$ noise in the input signal and the QPC is filtered out. To see this we start by recasting the problem in terms of the following dimensionless parameters

$$
\begin{aligned}
& x(t) \equiv \sqrt{\frac{1}{\hbar Z_{\mathrm{LC}}}}\left[\Phi(t)-\Phi_{\mathrm{ss}}\right] \equiv \alpha_{\mathrm{x}}\left[\Phi(t)-\Phi_{\mathrm{ss}}\right], \\
& y(t) \equiv \sqrt{\frac{Z_{\mathrm{LC}}}{\hbar}}\left[Q(t)-Q_{\mathrm{ss}}\right] \equiv \alpha_{\mathrm{y}}\left[Q(t)-Q_{\mathrm{ss}}\right],
\end{aligned}
$$

where $\alpha_{\mathrm{x}}$ and $\alpha_{\mathrm{y}}$ are implicitly defined above and the subscript ss refers to the steady state. The resulting equations are

$$
\begin{aligned}
& \dot{x}(t)=-\gamma x(t)+\omega_{0} y(t)-2 \alpha_{\mathrm{x}}\left[V_{\mathrm{c}}(t)+N(t)\right], \\
& \dot{y}(t)=-\omega_{0} x(t)-\alpha_{\mathrm{y}} \tilde{I}(t),
\end{aligned}
$$


where $\tilde{I}(t)=I(t)-I_{\mathrm{ss}}$. The solution of these coupled equations involves the two time-scales $\omega_{0}^{-1}$ and $\gamma^{-1}$. In the limit $\gamma^{-1} \gg \omega_{0}^{-1}(Q \gg 1)$, we can define a coarsegraining 50 time-scale $\mathrm{d} t$ (Roman font $\mathrm{d}$ ) that is short compared to $\gamma^{-1}$, and long compared to $\omega_{0}^{-1}$. On this time-scale we can make the standard rotating wave approximation (RWA), this being terms oscillating at frequencies greater than or equal to $\omega_{0}$ take their average value (unless they multiply white noise terms, that have non-negligible components at $\left.\omega_{0}\right)$.

Applying the above to Eqs. (16) gives

$$
\begin{aligned}
& \mathrm{d} \tilde{x}(t)=\left[-\frac{\gamma}{2} \tilde{x}(t)-\alpha_{\mathrm{x}} V_{\mathrm{in}}^{\mathrm{rf}}+\alpha_{\mathrm{y}} \tilde{I}^{\sin }(t)\right] \mathrm{d} t, \\
& \mathrm{~d} \tilde{y}(t)=\left[-\frac{\gamma}{2} \tilde{y}(t)-\alpha_{\mathrm{y}} \tilde{I}^{\cos }(t)\right] \mathrm{d} t,
\end{aligned}
$$

where the tilde denotes that we are in the frame rotating at $\omega_{0}$ and we have dropped the effect of the input noise, $N(t)$, as we assume that it is mainly $1 / f$ noise and has negligible spectral weight at $\omega_{0}$. The two currents $\tilde{I}^{\cos }(t)$ and $\tilde{I}^{\sin }(t)$ are the two quadratures of the filtered detector current $\tilde{I}(t)$ centered at the frequency $\omega_{0}$. That is, they are given by

$$
\begin{aligned}
\tilde{I}^{\cos }(t) & \equiv \frac{1}{\mathrm{~d} t} \int_{t-\mathrm{d} t}^{t} \tilde{I}(s) \cos \left(\omega_{0} s\right) \mathrm{d} s \\
\tilde{I}^{\sin }(t) & \equiv \frac{1}{\mathrm{~d} t} \int_{t-\mathrm{d} t}^{t} \tilde{I}(s) \sin \left(\omega_{0} s\right) \mathrm{d} s .
\end{aligned}
$$

These components are band-pass-filtered currents over the bandwidth $\mathrm{d} t^{-1}$ and are the quantities to which an idealized observer would have access. To be more specific, the current coming from the QPC is

$$
\tilde{I}(t)=e|\mathcal{T}| J(t)+\tilde{I}_{\text {clas }}(t),
$$

where $J(t)$ is the quantum signal given by

$$
J(t)=\sqrt{\kappa_{0}(t)}\left\langle\hat{\sigma}_{z}\right\rangle+\xi(t) .
$$

where the rate $\kappa_{0}$ will be discussed later, and $\xi(t)$ is the QPC shot noise, which we take to be white in the limit of a weakly responding QPC 9,10 That is, the QPC shot noise is Gaussian noise with the following correlations

$$
\begin{aligned}
\mathrm{E}[\xi(t)] & =0 \\
\mathrm{E}\left[\xi(t) \xi\left(t^{\prime}\right)\right] & =\delta\left(t-t^{\prime}\right) .
\end{aligned}
$$

Here E denotes an ensemble average over all possible realizations of $\xi(t)$. In Eq. (19) $\tilde{I}_{\text {clas }}(t)$ is the deterministic classical component of the current (with the steady-state current subtracted — see Appendix A for details). Using Eq. (13) we can write

$$
|\mathcal{T}(t)| \approx\left|\mathcal{T}_{0}\right|\left[1+\frac{1}{2} \epsilon_{\mathrm{in}} \sin \left(\omega_{0} t\right)\right]
$$

since $\epsilon_{\text {in }} \ll 1$ (see Sec. IIIA). Using this, the two quadrature currents become

$$
\begin{gathered}
\tilde{I}^{\cos }(t)=e\left|\mathcal{T}_{0}\right| \sqrt{S^{\cos }} J^{\cos }(t)+\tilde{I}_{\text {clas }}^{\cos }(t), \\
\tilde{I}^{\sin }(t)=e\left|\mathcal{T}_{0}\right| \sqrt{S^{\sin }} J^{\sin }(t)+\tilde{I}_{\text {clas }}^{\sin }(t),
\end{gathered}
$$

where $J^{\cos }(t)$ and $J^{\sin }(t)$ are the two quadrature components of $J(t)$. They are

$$
\begin{aligned}
& J^{\cos }(t)=\frac{1}{\sqrt{S^{\cos }}}\left[J_{\omega_{0}}^{\cos }(t)+\frac{1}{4} \epsilon_{\mathrm{in}} J_{2 \omega_{0}}^{\sin }(t)\right], \\
& J^{\sin }(t)=\frac{1}{\sqrt{S^{\sin }}}\left[J_{\omega_{0}}^{\sin }(t)+\frac{1}{4} \epsilon_{\mathrm{in}}\left[J_{0}^{\cos }(t)-J_{2 \omega_{0}}^{\cos }(t)\right]\right]
\end{aligned}
$$

where $S^{\cos }$ and $S^{\text {sin }}$ are dimensionless and defined such that $\left[J^{\cos }(t) \mathrm{d} t\right]^{2}=\left[J^{\sin }(t) \mathrm{d} t\right]^{2}=\mathrm{d} t$. The Fourier components of the quantum signal $J(t) \mathrm{d} t$ are

$$
\begin{aligned}
& J_{\mathrm{n} \omega_{0}}^{\cos }(t)=\frac{1}{\mathrm{~d} t} \int_{t-\mathrm{d} t}^{t} J(s) \cos \left(n \omega_{0} s\right) \mathrm{d} s, \\
& J_{\mathrm{n} \omega_{0}}^{\sin }(t)=\frac{1}{\mathrm{~d} t} \int_{t-\mathrm{d} t}^{t} J(s) \sin \left(n \omega_{0} s\right) \mathrm{d} s .
\end{aligned}
$$

Note that $\left[J_{\mathrm{n} \omega_{0}}^{\sin }(t) \mathrm{d} t\right]^{2}=\left[J_{\mathrm{n} \omega_{0}}^{\cos }(t) \mathrm{d} t\right]^{2}=\mathrm{d} t / 2$ for $n=1,2$; and that $\left[J_{0}^{\cos }(t) \mathrm{d} t\right]^{2}=\mathrm{d} t$ [using Eq. (21)].

To measure the two quadrature currents in Eq. (23) [and hence the quantum signals $J^{\cos }(t)$ and $\left.J^{\sin }(t)\right]$, an ideal observer would measure the desired quadrature of the output voltage. This can be done by beating the output voltage with a local oscillator $\stackrel{51}{=}$ Using Eq. (14c), the two output voltage quadratures are

$$
\begin{aligned}
& V_{\text {out }}^{\cos }(t)=\frac{B_{\sin }}{\alpha_{\mathrm{x}}} \frac{\gamma}{2} \int_{0}^{t} J^{\sin }(s) e^{-\gamma(t-s) / 2} d s, \\
& V_{\text {out }}^{\sin }(t)=-\frac{B_{\cos }}{\alpha_{\mathrm{x}}} \frac{\gamma}{2} \int_{0}^{t} J^{\cos }(s) e^{-\gamma(t-s) / 2} d s,
\end{aligned}
$$

where $B_{\text {sin }}=e\left|\mathcal{T}_{0}\right| \alpha_{\mathrm{y}} \sqrt{S^{\sin }}$, and $B_{\cos }=e\left|\mathcal{T}_{0}\right| \alpha_{\mathrm{y}} \sqrt{S^{\cos }}$ have units of $t^{-1 / 2}$. That is, $B_{\text {sin }}^{2}$ is the proportionality constant that turns the quantum signal, $J(t)$, into the dimensionless current $\alpha_{\mathrm{y}} \widetilde{I}^{\text {sin }}$, which drives the classical circuit (the same is also true for the cosine quadrature component). Here we have done some post-processing, removing the deterministic parts of $V_{\text {out }}^{\cos }(t)$ and $V_{\text {out }}^{\sin }(t)$, and dropping all contributions from $N(t)$ as again we assume that it has negligible spectral weight at $\omega_{0}$. By inverting these convolutions, the idealized observer can access both $J^{\cos }(t)$ and $J^{\sin }(t)$, enabling them to condition the quantum state on these currents. The resulting equation describing the idealized conditional dynamics of the qubit is called a stochastic master equation, or quantum trajectory equation. Note that we can write Eq. (26a) as $V_{\text {out }}^{\cos }(t)=\gamma \tilde{x}(t) /\left(2 \alpha_{\mathrm{x}}\right)$ (after some post processing), and similarly for Eq. (26b) in terms of $\tilde{y}$.

\section{IDEALIZED QUANTUM DYNAMICS}

We now consider the idealized case where the stochastic QPC current can be perfectly measured [we have access to both $J^{\cos }(t)$ and $\left.J^{\sin }(t)\right]$. To describe the idealized conditional qubit dynamics, we start with the microscopic model of charge qubit monitoring by a (dc) 
QPC in Ref. 12. We make an important modification to the model that is due to the time-dependence of the voltage across the rf-QPC, $V_{\mathrm{d}}(t)$ [see Eq. (13)]. It results in time-dependent QPC tunnelling rates. We also make a rotating wave approximation (RWA) to simplify the analysis. The RWA is only possible for a weakly responding QPC. In this paper, as in Ref. 12, we will refer to the limit of weak response as quantum diffusion. This is because in this limit there are many electrons passing through the QPC with each containing only a little information about the qubit state, which under monitoring of the QPC current the evolution of the qubit will slowly wander towards one of the $\hat{\sigma}_{z}$ eigenstates rather then a sudden collapse. The linear form of the qubit stochastic master equation of Ref. 12 is (see Appendix $\mathrm{A}$ for details)

$$
\begin{aligned}
d \bar{\rho}_{\mathrm{c}}(t) \equiv & -\frac{i}{\hbar}\left[\hat{H}_{\mathrm{qb}}^{\prime}(t)+\hat{H}_{\mathrm{J}}(t), \bar{\rho}_{\mathrm{c}}(t)\right] d t \\
& +\frac{1}{2} \Gamma_{\mathrm{d}}(t) \mathcal{D}\left[\hat{\sigma}_{z}\right] \bar{\rho}_{\mathrm{c}}(t) d t \\
& +[J(t)-\mu] d t \overline{\mathcal{H}}\left[\sqrt{\kappa_{0}(t)} \hat{\sigma}_{z} / 2\right] \bar{\rho}_{\mathrm{c}}(t),
\end{aligned}
$$

where

$$
\begin{aligned}
\hat{H}_{\mathrm{qb}}^{\prime}(t) & =\hat{H}_{\mathrm{qb}}+\hbar \hat{\sigma}_{z}|\mathcal{T}(t)||\mathcal{X}(t)| \sin (\theta) / 2, \\
\hat{\bar{H}}_{\mathrm{J}}(t) & =-\hbar \hat{\sigma}_{z}[J(t)-\mu] \sqrt{\kappa_{1}(t)} / 2,
\end{aligned}
$$

and $\mu$ is the mean of the ostensible distribution used to derive Eq. (27) (see Appendix A). The linear superoperator, $\overline{\mathcal{H}}$, in Eq. (27) is defined for arbitrary operators $\hat{c}$ by

$$
\overline{\mathcal{H}}[\hat{c}] \rho=\hat{c} \rho+\rho \hat{c}^{\dagger}-\mu \rho .
$$

It represents the back action effects of the continuous measurement. The time-dependent qubit dephasing rate is $\Gamma_{\mathrm{d}}(t)=\left[\kappa_{0}(t)+\kappa_{1}(t)\right] / 2$, where $\kappa_{0}(t)$ and $\kappa_{1}(t)$ represent two types of measurement-induced dephasing in the qubit: $\kappa_{0}(t)$ represents information-limited (Heisenberg) dephasing $\stackrel{15}{=}$ which reflects the quantum-mechanical cost of obtaining information about the qubit state; $\kappa_{1}(t)$ represents additional (non-Heisenberg) dephasing by processes that yield no qubit information. We define these dephasing rates by

$$
\begin{aligned}
& \sqrt{\kappa_{0}(t)} \equiv|\mathcal{X}(t)| \cos (\theta)=\sqrt{2 \Gamma_{\mathrm{d}}(t)} \cos (\theta), \\
& \sqrt{\kappa_{1}(t)} \equiv|\mathcal{X}(t)| \sin (\theta)=\sqrt{2 \Gamma_{\mathrm{d}}(t)} \sin (\theta),
\end{aligned}
$$

where $\theta$ is the relative phase between the QPC tunnelling amplitudes $\mathcal{T}$ and $\mathcal{X}$. Note that if $\theta=0$ or $\theta=\pi$, then $\kappa_{1}=0$, and $\hat{\bar{H}}_{\mathrm{J}}(t)=0$. This is a necessary condition for the QPC to be considered an ideal charge qubit detector operating at the quantum limit. $9,52,53$

From Eq. (13) we can write

$$
|\mathcal{X}(t)| \approx \sqrt{\kappa}\left[1+\frac{1}{2} \epsilon_{\mathrm{in}} \sin \left(\omega_{0} t\right)\right],
$$

since $\epsilon_{\text {in }} \ll 1$ (see Sec. IIIA). Here the time-dependence has been made explicit, and $\kappa / 2=|\mathcal{X}|^{2} / 2$ is the timeindependent dephasing rate for the charge qubit monitored by a dc-QPC (the situation in Ref. 12). Substituting $\kappa_{0}(t)$ and $\kappa_{1}(t)$ (with the above approximation for their time dependence) into Eq. (27) and making the RWA gives

$$
\begin{aligned}
\mathrm{d} \bar{\rho}_{\mathrm{c}}(t)= & -\frac{i}{\hbar}\left[\widetilde{H}_{\mathrm{qb}}+\widetilde{H}_{\mathrm{J}}(t), \bar{\rho}_{\mathrm{c}}(t)\right] \mathrm{d} t \\
+ & \kappa \mathcal{D}\left[\hat{\sigma}_{z}\right] \bar{\rho}_{\mathrm{c}}(t) \mathrm{d} t / 4 \\
+ & \left\{J_{0}^{\cos }(t)-\mu+\frac{\epsilon_{\mathrm{in}}}{2}\left[J_{\omega_{0}}^{\sin }(t)-\mu\right]\right\} \mathrm{d} t \\
& \times \overline{\mathcal{H}}\left[\sqrt{\kappa} \cos (\theta) \hat{\sigma}_{z} / 2\right] \bar{\rho}_{\mathrm{c}}(t),
\end{aligned}
$$

where $J_{0}^{\cos }(t)$ and $J_{\omega_{0}}^{\sin }(t)$ are defined in Eqs. (25). The rotated versions of the Hamiltonians are

$$
\begin{aligned}
\widetilde{H}_{\mathrm{J}}(t)= & -\hbar \hat{\sigma}_{z}\left\{J_{0}^{\cos }(t)-\mu+\frac{\epsilon_{\mathrm{in}}}{2}\left[J_{\omega_{0}}^{\sin }(t)-\mu\right]\right\} \\
& \times \sqrt{\kappa} \sin (\theta) / 2, \\
\widetilde{H}_{\mathrm{qb}} \approx & \hat{H}_{\mathrm{qb}}+\hbar \hat{\sigma}_{z}\left|\mathcal{T}_{0}\right| \sqrt{\kappa} \sin (\theta) / 2 .
\end{aligned}
$$

To get the linear form of the ideal quantum trajectory we need to rewrite Eq. (32) in terms of the signal that an ideal observer could access, namely $J^{\sin }(t)$. This is achieved by expressing $J_{0}^{\cos }(t)$ and $J_{\omega_{0}}^{\sin }(t)$ in terms of both the observed process, $J^{\sin }(t)$, plus some other unobserved processes, as done in Ref. 21. Averaging over the unobserved processes results in the following linear quantum trajectory equation in the RWA:

$$
\begin{aligned}
\mathrm{d} \bar{\rho}_{\mathrm{c}}(t)= & \tilde{\mathcal{L}} \bar{\rho}_{\mathrm{c}}(t) \mathrm{d} t \\
& +\sqrt{\eta}\left[J^{\sin }(t)-\sqrt{\eta} \mu\right] \mathrm{d} t \overline{\mathcal{H}}\left[\sqrt{\kappa} \hat{\sigma}_{z} / 2\right] \bar{\rho}_{\mathrm{c}}(t),
\end{aligned}
$$

where we have defined the efficiency $\eta$ by

$$
\eta \equiv \epsilon_{\mathrm{in}}^{2} \cos ^{2}(\theta) S^{\mathrm{sin}} \approx \epsilon_{\mathrm{in}}^{2} \cos ^{2}(\theta) / 4,
$$

and the Liouvillian $\tilde{\mathcal{L}}$ is

$$
\tilde{\mathcal{L}} \rho=-\frac{i}{\hbar}\left[\widetilde{H}_{\mathrm{qb}}+\widetilde{H}_{\mathrm{J}}^{\prime}(t), \rho\right]+\frac{\kappa}{4} \mathcal{D}\left[\hat{\sigma}_{z}\right] \rho .
$$

We now have $\widetilde{H}_{\mathrm{J}}^{\prime}(t)=-\hbar \hat{\sigma}_{z} J^{\sin }(t) \sqrt{\eta \kappa} \tan (\theta) / 2$. Equation (34) normalizes to

$$
\begin{aligned}
\mathrm{d} \rho_{\mathrm{c}}(t)=\tilde{\mathcal{L}} \rho_{\mathrm{c}} \mathrm{d} t+ & \sqrt{\eta}\left[J^{\sin }(t)-\sqrt{\eta \kappa}\left\langle\hat{\sigma}_{z}\right\rangle\right] \mathrm{d} t \\
& \times \mathcal{H}\left[\sqrt{\kappa} \hat{\sigma}_{z} / 2\right] \rho_{\mathrm{c}}(t),
\end{aligned}
$$

where the normalized current is

$$
J^{\sin }(t) \mathrm{d} t=\sqrt{\eta \kappa}\left\langle\hat{\sigma}_{z}\right\rangle \mathrm{d} t+\mathrm{d} W(t)
$$

where $\mathrm{d} W(t)=\xi(t) \mathrm{d} t$ is a Wiener increment $\underline{54}$ This is the explicit expression for the quantum signal to which an ideal observer would have direct access. To get the correct statistics, we have made a Girsanov transformation, $\sqrt{55}$ which results in replacing $\mu$ in the above with $\sqrt{\kappa}\left\langle\hat{\sigma}_{z}\right\rangle$. The stochastic Hamiltonian $\widetilde{H}_{\mathrm{J}}^{\prime}(t)$ in Eq. (36) will also be updated in the same manner (replacing $\mu$ with $\left.\sqrt{\kappa}\left\langle\hat{\sigma}_{z}\right\rangle\right)$. The nonlinear back action superoperator is defined by its action on $\rho$ with an arbitrary operator $\hat{c}$ by

$$
\mathcal{H}[\hat{c}] \rho=\hat{c} \rho+\rho \hat{c}^{\dagger}-\left\langle\hat{c}+\hat{c}^{\dagger}\right\rangle \rho .
$$


Equation (37) is the first stochastic master equation presented for continuous measurement of a charge qubit using an rf configuration. It reveals some interesting physics about the rf-QPC operating in the $\mathrm{rf}+\mathrm{dc}$ mode. First, we note that the qubit dephasing is due only to the dc component of the detector voltage, since $\kappa$ is a function of $V_{0}$ [and is independent of the $\mathrm{AC}$ component of $\left.V_{\mathrm{d}}(t)\right]$. Second, the rf-QPC is a highly inefficient detector when operated in the $\mathrm{rf}+\mathrm{dc}$ mode since $\eta \propto \epsilon_{\text {in }}^{2} \ll 1$. Physically, this low detection efficiency arises because the qubit information is extracted relatively slowly by the (small) rf component of the QPC voltage, compared to the qubit dephasing by the (large) dc component. We conjecture that the low measurement efficiency of the $\mathrm{rf}+\mathrm{dc}$ QPC may in practice be higher than that of the dc-QPC for two reasons: $1 / f$ noise limits the dc-QPC measurement efficiency and also further degrades the efficiency the longer the measurement has to continue.

\section{REALISTIC DYNAMICS: REALISTIC QUANTUM TRAJECTORY EQUATION}

The previous sections conditioned the qubit evolution and the classical oscillator evolution on idealized measurement results available only to a hypothetical observer. For an experimentalist, it is much more useful to consider how to describe the qubit evolution conditioned on measurement results available in the laboratory. In rf-QPC or rf-SET experiments, the voltage leaving the transmission line is observed using homodyne detection of the amplitude quadrature $(\tilde{x})$. The phase quadrature $(\tilde{y})$ can be ignored because it is independent of the qubitinformation-carrying signal, $J^{\sin }(t)$ [see Eqs. (17) and (26)].

In simple dyne detection (see schematic in Fig. 2), the output signal $V_{\text {out }}(t)$ is amplified, and mixed with a local oscillator (LO). The LO for homodyne detection of the amplitude quadrature is $V_{\mathrm{LO}}(t) \propto \cos \left(\omega_{0} t\right)$, where the LO frequency is the same as the signal of interest (or very slightly detuned). The resulting low-frequency beats due to mixing the signal with the LO are easily detected.

The signal resulting from the homodyne detection is

$$
\begin{aligned}
\mathcal{V}(t) \mathrm{d} t & =\sqrt{A_{\mathrm{SN}}} \frac{\alpha_{\mathrm{x}}}{B_{\text {sin }}} V_{\text {out }}^{\cos }(\tilde{x}) \mathrm{d} t+\mathrm{d} W_{\text {out }}(t) \\
& \equiv \lambda_{\tilde{x}} \mathrm{~d} t+\mathrm{d} W_{\text {out }}(t),
\end{aligned}
$$

where $A_{\mathrm{SN}}$ is the ratio of gain squared to noise power which has the dimensions of inverse time. This relates to the dimensionless signal-to-noise ratio for the measurement as $\mathrm{SNR}=A_{\mathrm{SN}} t$ ( $t$ is the measurement duration). Equation (40) shows that white noise is added to the amplified quadrature signal. Here

$$
\lambda_{\tilde{x}} \equiv \sqrt{A_{\mathrm{SN}}} \frac{\gamma \tilde{x}}{2 B_{\mathrm{sin}}},
$$

and the output noise Wiener increment $\mathrm{d} W_{\text {out }}(t)$ satisfies $\left[\mathrm{d} W_{\text {out }}(t)\right]^{2}=\mathrm{d} t$. The addition of this extra noise pre- vents one from inverting the convolutions in Eqs. (26) to find $J^{\sin }(t)$, and so the realistic quantum trajectory approach 22,23 must be employed to condition the qubit evolution on the realistic measurement record $\mathcal{V}(t)$.

Our derivation of the realistic quantum trajectory equation describing the conditional evolution of the combined oscillator-qubit state closely follows the standard techniques. 21,23 The details are included in Appendix B. with the final result being the following superoperator Kushner-Stratonovich (SKS) equation:

$$
\begin{aligned}
\mathrm{d} \rho_{\mathcal{V}}(\tilde{x})= & \left\{\left[-\frac{\partial}{\partial \tilde{x}} m_{\tilde{x}}+\frac{B_{\sin }^{2}}{2} \frac{\partial^{2}}{\partial \tilde{x}^{2}}+\tilde{\mathcal{L}}\right] \mathrm{d} t\right. \\
& \left.+\left(\lambda_{\tilde{x}}-\lambda_{\langle\tilde{x}\rangle_{\rho}}\right)\left[\mathcal{V}(t)-\lambda_{\langle\tilde{x}\rangle_{\rho}}\right] \mathrm{d} t\right\} \rho_{\mathcal{V}}(\tilde{x}) \\
& -\mathrm{d} t \sqrt{\eta \kappa} B_{\sin } \frac{\partial}{\partial \tilde{x}}\left[\hat{\sigma}_{z} \rho_{\mathcal{V}}(\tilde{x})+\rho_{\mathcal{V}}(\tilde{x}) \hat{\sigma}_{z}\right] / 2,
\end{aligned}
$$

where we now consider $\tilde{x}$ to be post-processed (the deterministic part has been removed), and we have defined $m_{\tilde{x}} \equiv-\gamma \tilde{x} / 2$. The true record the realistic observer would measure is

$$
\mathcal{V}(t) \mathrm{d} t=\lambda_{\langle\tilde{x}\rangle_{\rho}} \mathrm{d} t+\mathrm{d} \mathcal{W}(t)
$$

where the observed white noise $\mathrm{d} \mathcal{W}(t)$ is not the same as $\mathrm{d} W_{\text {out }}(t)$ [contrast (43) and (40)]. In Eq. (42), the Liouvillian superoperator $\mathcal{L}$ contains the Hamiltonian evolution and qubit dephasing (and is defined in Appendix B). The realistic quantum trajectory equation (42) is an important result of this paper. The first line describes the uncoupled, average evolution of the oscillator and the qubit. The second line describes the update of the realistic observer's state of knowledge of the supersystem, conditioned on the realistic homodyne output. The final line contains the effect of the qubit on the circuit.

The (reduced) state of the qubit conditioned on the realistic output, $\rho_{\mathcal{V}}$, is found from $\rho_{\mathcal{V}}(\tilde{x})$ by integrating out the circuit variable $\tilde{x}$. Similarly, the (marginal) state of the circuit can be found by tracing out the qubit.

\section{OSCILLATOR SLAVED TO QUBIT}

For sufficiently large damping $\gamma$ such that the first term in Eq. 17a dominates the dynamics of $\tilde{x}$ (but still $\gamma_{\mathrm{d}} \gg \gamma$, so that the RWA remains valid), the oscillator immediately damps to a qubit-dependent state. The circuit is thus said to be slaved to the qubit. This adiabatic elimination of the circuit allows the conditioned qubit dynamics to be once again governed by the stochastic master equation alone. However, a realistic observer has access only to the homodyne output, that involves excess noise above that of $J^{\sin }$. Thus, we find the slaved output to which the realistic observer has access, then use it to condition the qubit state. The slaved value of $\tilde{x}$ is found 
by taking $\gamma \rightarrow \infty$ in Eq. 26a . This allows us to make the standard delta-function replacement and write the quadrature output voltage as $V_{\text {out }}^{\cos }(t)=B_{\sin } J^{\sin }(t) / \alpha_{\mathrm{x}}$. That is, the quadrature output voltage is directly proportional to the quantum signal an ideal observer would measure. Substituting this into Eq. (40) gives

$$
\mathcal{V}_{\mathrm{sl}}(t) \mathrm{d} t=\sqrt{A_{\mathrm{SN}}} J^{\sin }(t) \mathrm{d} t+\mathrm{d} W_{\text {out }}(t),
$$

where $J^{\sin }(t)$ is the signal used to condition the qubit state in Eq. (34). Here we see that the output noise degrades the QPC signal, the effect of which is to reduce the efficiency of the detection.

Using the above we can redefine the quantum signal a realistic observer would measure as $\mathcal{V}_{\mathrm{sl}}(t)=$ $J_{\mathrm{sl}}(t) \sqrt{A_{\mathrm{SN}}}+1$, where

$$
J_{\mathrm{sl}}(t) \mathrm{d} t=\sqrt{\eta_{\mathrm{sl}} \kappa}\left\langle\hat{\sigma}_{z}\right\rangle \mathrm{d} t+\mathrm{d} \mathcal{W}(t)
$$

where

$$
\eta_{\mathrm{sl}}=\eta \frac{A_{\mathrm{SN}}}{A_{\mathrm{SN}}+1}
$$

is the efficiency of the realistic $\mathrm{rf}+\mathrm{dc} \mathrm{QPC}$ in the slaved limit. Using this quantum signal, the stochastic master equation in the slaved limit is

$$
\begin{aligned}
\mathrm{d} \rho_{\mathrm{c}}(t)=\mathrm{d} t \tilde{\mathcal{L}} \rho_{\mathrm{c}}(t)+\sqrt{\eta_{\mathrm{sl}}}\left[J_{\mathrm{sl}}(t)-\sqrt{\eta_{\mathrm{sl}} \kappa}\left\langle\hat{\sigma}_{z}\right\rangle\right] \mathrm{d} t \\
\times \mathcal{H}\left[\sqrt{\kappa} \hat{\sigma}_{z} / 2\right] \rho_{\mathrm{c}}(t),
\end{aligned}
$$

where the noise term in $\tilde{\mathcal{L}}$ is now in terms of $J_{\mathrm{sl}}(t)$. The fact that the efficiency $\eta_{\mathrm{sl}}$ in Eq. (46) is strictly less than unity shows that the $\mathrm{rf}+\mathrm{dc} \mathrm{QPC}$ is incapable of reaching the quantum limit of a purity-preserving detector, even in the slaved limit. This conclusion is expected from the discussion following the idealized stochastic master equation in the RWA, Eq. (37). That is, even without including the circuit in our description of the rf-QPC, Eq. (37) shows that, for $\epsilon_{\text {in }} \ll 1$ (the rf + dc mode), the rf-QPC is a highly inefficient charge qubit detector (that may nonetheless be more efficient than the dc-QPC due to the effects of $1 / f$ noise in the dc case). Note that the theoretical maximum of $\eta_{\mathrm{sl}}$ is the idealized efficiency $\eta$, which occurs for infinite signal-to-noise ratio in the amplifier. As a final point, we note that the inclusion of input white noise in the analysis will have the effect of further reducing the $\mathrm{rf}+\mathrm{dc}$ QPC efficiency (and significantly complicating the analysis) $\underline{\underline{56}}$

\section{DISCUSSION AND SUMMARY}

In this paper we have presented a model for conditional monitoring of a charge qubit using an rf (radiofrequency) configuration. The rf configuration was introduced for the single-electron transistor (SET) in Ref. 26. It involves embedding the SET in a resonant (oscillator) circuit, and monitoring the resulting damping. This allows operation of the SET at high frequencies, where the $1 / f$ noise prevalent in conventional low-frequency, $\mathrm{dc}$ measurements is completely negligible.

We have two main results. We have derived an evolution equation for the conditional state of a charge qubit monitored continuously in time by a detector operated in the rf configuration. To the best of our knowledge, an equation of this type has not previously been derived. This culminates in the stochastic master equation, or quantum trajectory, Eq. (37). Our second main result is the extension of our idealized quantum trajectory (37) to consider conditioning the qubit state on a corrupted (filtered, more noisy) measurement signal available to a realistic observer. Our realistic quantum trajectory $21,22,23$ equation is Eq. (42).

Our model used the quantum-point contact (QPC) in place of the SET, and also assumed operation of the rfQPC in the so-called ${ }^{44} \mathrm{rf}+\mathrm{dc}$ mode. In this mode, a small amplitude sinusoid (carrier) at the resonance frequency of the oscillator is superposed on a relatively large bias (dc). Experimentally $\stackrel{44}{\underline{4}}$ this is done to maximize the sensitivity of a SET. In this mode we can make a rotating wave approximation (RWA) in the limit of a vanishingly small amplitude sinusoid. We found the rf + dc QPC to be a highly inefficient charge-qubit detector, even ignoring the external circuit. Physically, this is because the small rf amplitude carries the qubit information, while the relatively large dc bias causes most of the measurementinduced qubit dephasing. It is important to note that the low measurement efficiency of the $\mathrm{rf}+\mathrm{dc}$ QPC may in practice be higher than for the dc-QPC due to the debilitating effects of $1 / f$ noise. Specifically, $1 / f$ noise limits the dc-QPC measurement efficiency, and also further degrades the efficiency the longer the measurement has to continue.

Having realistically modeled continuous-in-time qubit measurement using the $\mathrm{rf}+\mathrm{dc} \mathrm{QPC}$, the next step is to extend realistic quantum trajectory theory to the pure rf mode (zero bias, larger rf amplitude) for both the QPC and the SET. These are important future tasks, particularly for understanding and designing measurement and feedback control of quantum systems in the solid-state valuable knowledge for fully harnessing the potential of future quantum technologies such as quantum computers.

\section{Acknowledgments}

This work was supported by the ARC and the State of Queensland. NO acknowledges partial support from the QIP IRC and QAP. JG was partially supported by MITACS and ORDCF. 


\section{APPENDIX A: RECASTING THE STOCHASTIC MASTER EQUATION OF GOAN, ET AL.}

The microscopic model of charge qubit monitoring by a conventional (dc) QPC in Ref. 12 is used in this appendix as a starting point for producing a stochastic master equation for the rf-QPC (in the $\mathrm{rf}+\mathrm{dc}$ mode) [Eq. 27. We add time dependence to the QPC tunnelling rates that is due to the time-dependent rf-QPC voltage (see Sec. IIIA), and recast the diffusive stochastic master equation of Ref. 12 into a linear form.

The diffusive stochastic master equation of Ref. 12 is

$$
\begin{aligned}
d \rho_{\mathrm{c}}(t)= & -\frac{i}{\hbar}\left[\hat{H}_{\mathrm{qb}}, \rho_{\mathrm{c}}(t)\right] d t+\mathcal{D}[\mathcal{T}+\mathcal{X} \hat{n}] \rho_{\mathrm{c}}(t) d t \\
& +\xi(t) d t \frac{1}{|\mathcal{T}|}\left[\mathcal{T}^{*} \mathcal{X} \hat{n} \rho_{\mathrm{c}}(t)+\mathcal{X}^{*} \mathcal{T} \rho_{\mathrm{c}}(t) \hat{n}\right] \\
& -\xi(t) d t \frac{1}{|\mathcal{T}|} 2 \operatorname{Re}\left(\mathcal{T}^{*} \mathcal{X}\right)\langle\hat{n}\rangle_{\mathrm{c}} \rho_{\mathrm{c}}(t)
\end{aligned}
$$

where $\hat{n}=|1\rangle\langle 1|$ and $\hat{H}_{\mathrm{qb}}$, and the time-dependencies of $\mathcal{T}(t)$ and $\mathcal{X}(t)$ in our model are defined in Sec. III. This equation can be rewritten as

$$
\begin{aligned}
d \rho_{\mathrm{c}}(t)= & -\frac{i}{\hbar}\left[\hat{H}_{\mathrm{qb}}^{\prime}(t), \rho_{\mathrm{c}}(t)\right] d t+\frac{1}{2} \Gamma_{\mathrm{d}}(t) \mathcal{D}\left[\hat{\sigma}_{z}\right] \rho_{\mathrm{c}}(t) d t \\
+ & \xi(t) d t\left\{\mathcal{H}\left[\sqrt{\kappa_{0}(t)} \hat{\sigma}_{z} / 2\right]\right. \\
& \left.+\mathcal{H}\left[\sqrt{\kappa_{1}(t)} i \hat{\sigma}_{z} / 2\right]\right\} \rho_{\mathrm{c}}(t)
\end{aligned}
$$

where

$$
\begin{aligned}
\hat{H}_{\mathrm{qb}}^{\prime}(t) & =\hat{H}_{\mathrm{qb}}+\hbar \hat{\sigma}_{z}|\mathcal{T}(t)||\mathcal{X}(t)| \sin (\theta) / 2, \\
\sqrt{\kappa_{0}(t)} & =|\mathcal{X}(t)| \cos (\theta), \\
\sqrt{\kappa_{1}(t)} & =|\mathcal{X}(t)| \sin (\theta), \\
\Gamma_{\mathrm{d}}(t) & =\frac{\kappa_{0}(t)+\kappa_{1}(t)}{2}=\frac{|\mathcal{X}(t)|^{2}}{2} .
\end{aligned}
$$

Here $\theta=\arg \left(\mathcal{T}^{*} \mathcal{X}\right)$ is the relative phase between $\mathcal{T}(t)$ and $\mathcal{X}(t)$. The superoperator $\mathcal{H}[\hat{c}]$ in Eq. (A2) is defined by its action on $\rho$ in Eq. (39).

In Ref. 12 the current through the QPC is

$$
I(t)=e\left\{|\mathcal{T}|^{2}+2 \operatorname{Re}\left[\mathcal{T}^{*} \mathcal{X}\right]\langle\hat{n}\rangle+|\mathcal{T}| \xi(t)\right\},
$$

which in our parameterization is (now with timedependent $\mathcal{T}$ and $\mathcal{X}$ )

$$
I(t)=e|\mathcal{T}(t)|\left[|\mathcal{T}(t)|+\sqrt{\kappa_{0}(t)}\left(1+\left\langle\hat{\sigma}_{z}\right\rangle\right)+\xi(t)\right] .
$$

Here we see that this current comprises two parts: a quantum signal, $J(t)$, that depends on the state of the qubit and the noise $\xi(t)$; and a large deterministic classical signal, $I_{\text {clas }}(t)$. The quantum and classical signals are

$$
\begin{aligned}
J(t) & =\sqrt{\kappa_{0}(t)}\left\langle\hat{\sigma}_{z}\right\rangle+\xi(t), \\
I_{\text {clas }}(t) & =e|\mathcal{T}(t)|\left[\sqrt{\kappa_{0}(t)}+|\mathcal{T}(t)|\right],
\end{aligned}
$$

which allows us to write $I(t)$ as

$$
I(t)=e|\mathcal{T}(t)| J(t)+I_{\text {clas }}(t) .
$$

Using the quantum signal we can rewrite the stochastic master equation [Eq. (A-2)] as

$$
\begin{aligned}
d \rho_{\mathrm{c}}(t)= & -\frac{i}{\hbar}\left[\hat{H}_{\mathrm{qb}}^{\prime}(t)+\hat{H}_{\mathrm{J}}(t), \rho_{\mathrm{c}}(t)\right] d t \\
& +\frac{1}{2} \Gamma_{\mathrm{d}}(t) \mathcal{D}\left[\hat{\sigma}_{z}\right] \rho_{\mathrm{c}}(t) d t \\
& +\left[J(t)-\sqrt{\kappa_{0}(t)}\left\langle\hat{\sigma}_{z}\right\rangle\right] d t \mathcal{H}\left[\sqrt{\kappa_{0}(t)} \hat{\sigma}_{z} / 2\right] \rho_{\mathrm{c}}(t),
\end{aligned}
$$

where

$$
\hat{H}_{\mathrm{J}}(t)=-\hbar \hat{\sigma}_{z}\left[J(t)-\sqrt{\kappa_{0}(t)}\left\langle\hat{\sigma}_{z}\right\rangle\right] \sqrt{\kappa_{1}(t)} / 2 .
$$

Equation (A9) is the normalized quantum trajectory an observer would use to describe their state of knowledge of the qubit if they had access to the quantum signal $J(t)$.

To derive the realistic quantum trajectory, we use linear quantum trajectory theory, $, 2,29,57,58$ so we now recast the above in linear form. The linear quantum trajectory is derived in essentially the same way as Eq. (A1) was done in Ref. 12, except that one must use an ostensible distribution for $J$ rather then the real signal [Eq. A6. That is, the possible results $J d t$ at time $t$ are chosen from a Gaussian distribution with mean $\mu$ and variance $d t$. Here $\mu$ is an arbitrary ostensible parameter, with the only constraint that the ostensible distribution for $J$ must be non-zero when the true distribution is non-zero. We don't go through the calculation in detail, but the final result is equivalent to simply replacing $\sqrt{\kappa_{0}(t)}\left\langle\hat{\sigma}_{z}\right\rangle$ with $\mu$ in Eq. (A9). That is, the linear quantum trajectory is

$$
\begin{aligned}
d \bar{\rho}_{\mathrm{c}}(t)= & -\frac{i}{\hbar}\left[\hat{H}_{\mathrm{qb}}^{\prime}+\hat{\bar{H}}_{\mathrm{J}}(t), \bar{\rho}_{\mathrm{c}}(t)\right] d t \\
& +\frac{1}{2} \Gamma_{\mathrm{d}}(t) \mathcal{D}\left[\hat{\sigma}_{z}\right] \bar{\rho}_{\mathrm{c}}(t) d t \\
& +[J(t)-\mu] d t \overline{\mathcal{H}}\left[\sqrt{\kappa_{0}(t)} \hat{\sigma}_{z} / 2\right] \bar{\rho}_{\mathrm{c}}(t),
\end{aligned}
$$

where the linear measurement superoperator is

$$
\overline{\mathcal{H}}[\hat{c}] \rho=\hat{c} \rho+\rho \hat{c}^{\dagger}-\mu \rho,
$$

and

$$
\hat{\bar{H}}_{\mathrm{J}}(t)=-\hbar \hat{\sigma}_{z}[J(t)-\mu] \sqrt{\kappa_{1}(t)} / 2 .
$$

Equation (A11) is Eq. (27).

\section{APPENDIX B: DERIVATION OF THE REALISTIC QUANTUM TRAJECTORY EQUATION}

In this appendix we derive a realistic quantum trajectory equation for the rf-QPC-monitored charge qubit. The derivation closely follows the derivation presented in 
Ref. 21 for the conventional QPC. As discussed, we ignore the phase quadrature of the classical oscillator since it reveals no qubit information [see Equations (17)]. Therefore, we describe the imperfect knowledge of the oscillator by a probability distribution for the amplitude quadrature, $P(\tilde{x})$.

\section{Stochastic Fokker-Planck equation for the oscillator}

For notational simplicity, we express the Langevin equation for $\tilde{x}(t)$, Eq. (17a) (after the deterministic classical part has been removed), as

$$
\mathrm{d} \tilde{x}(t)=\left[m_{\tilde{x}}+B_{\sin } J^{\sin }(t)\right] \mathrm{d} t,
$$

where we have defined

$$
m_{\tilde{x}} \equiv-\frac{\gamma}{2} \tilde{x}(t) .
$$

Equation (B1) describes the evolution of $\tilde{x}(t)$ for perfect knowledge of $J^{\sin }(t)$. A realistic observer will not have direct access to the idealized quadrature current in Eq. (B1), so we find an equation for $P(\tilde{x})$ (see Refs. 23 and 21 for details of the procedure). The result is the stochastic Fokker-Planck equation:

$$
\begin{aligned}
\mathrm{d} P_{\mathrm{c}}(\tilde{x})=\mathrm{d} t\{ & -\frac{\partial}{\partial \tilde{x}}\left[m_{\tilde{x}}+B_{\sin } J^{\sin }(t)\right] \\
& \left.+\frac{B_{\sin }^{2}}{2} \frac{\partial^{2}}{\partial \tilde{x}^{2}}\right\} P(\tilde{x}),
\end{aligned}
$$

As expected, we have both deterministic and stochastic drift $(\partial / \partial \tilde{x})$ of $P(\tilde{x})$, as well as the diffusion $\left(\partial^{2} / \partial \tilde{x}^{2}\right)$ associated with the stochastic drift. We next consider conditioning $P(\tilde{x})$ on the homodyne measurement results.

\section{Zakai equation for the oscillator}

Following Ref. 21, we find the best estimate for $P(\tilde{x})$ conditioned upon the measurement result $\mathcal{V}$ using Bayesian analysis. Denoted $P_{\mathcal{V}}(\tilde{x})$, this estimate is

$$
\bar{P}_{\mathcal{V}}(\tilde{x})=\frac{P_{\tilde{x}}(\mathcal{V}) P(\tilde{x})}{\Lambda(\mathcal{V})}
$$

where the bar indicates an unnormalized distribution. The ostensible distribution $\Lambda(\mathcal{V})$ is a Gaussian distribution of arbitrary mean $\lambda$, and some variance $\mathrm{v}$ :

$$
\Lambda(\mathcal{V})=\frac{1}{\sqrt{2 \pi \mathrm{v}}} \exp \left[-\frac{(\mathcal{V}-\lambda)^{2}}{2 \mathrm{v}}\right] .
$$

That is, for the Zakai equation we consider the observed output $\mathcal{V}$ to be ostensibly Gaussian white noise of mean $\lambda$. We simplify the derivation by choosing $\lambda=0$, but note that the choice of ostensible mean (in $\Lambda$ ) in the Zakai equation is arbitrary (subject to the condition that $\Lambda(\mathcal{V})$ is nonzero when $P(\mathcal{V})$ is nonzero). Inspection of Eq. (40) shows that $P_{\tilde{x}}(\mathcal{V})$ is a Gaussian distribution of mean $\lambda_{\tilde{x}}$ and variance $\mathrm{v}=1 / \mathrm{d} t$. That is,

$$
P_{\tilde{x}}(\mathcal{V})=\frac{\sqrt{\mathrm{d} t}}{\sqrt{2 \pi}} \exp \left\{-\frac{\left[\mathcal{V}-\lambda_{\tilde{x}}\right]^{2} \mathrm{~d} t}{2}\right\},
$$

where $\lambda_{\tilde{x}}$ is defined in Eq. (41). The results above combine into Eq. (B4) to give the Zakai equation

$$
\bar{P}_{\mathcal{V}}(\tilde{x})=\left\{1+\mathcal{V} \mathrm{d} t \lambda_{\tilde{x}}\right\} P(\tilde{x}) .
$$

This is exactly analogous to the Zakai equations derived for the conventional (dc-mode) QPC in Ref. 21.

\section{Combined equation for the oscillator}

As in Ref. 21, we choose to condition on measurement results after microscopic processes, so that the combined conditional evolution of the oscillator is given by

$$
P(\tilde{x})+\mathrm{d} \bar{P}_{\mathcal{V}, \mathrm{c}}(\tilde{x})=\left\{1+\mathcal{V} \mathrm{d} t \lambda_{\tilde{x}}\right\}\left[P(\tilde{x})+\mathrm{d} P_{\mathrm{c}}(\tilde{x})\right],
$$

where $\mathrm{d} P_{\mathrm{c}}(\tilde{x})$ is given by the stochastic Fokker-Planck equation (B3). We find that

$$
\begin{aligned}
\mathrm{d} \bar{P}_{\mathcal{V}, \mathrm{c}}(\tilde{x})= & \mathrm{d} t\left\{-\frac{\partial}{\partial \tilde{x}}\left[m_{\tilde{x}}+B_{\sin } J^{\sin }(t)\right]+\frac{B_{\sin }^{2}}{2} \frac{\partial^{2}}{\partial \tilde{x}^{2}}\right. \\
& \left.+\mathcal{V} \lambda_{\tilde{x}}\right\} P(\tilde{x}) .
\end{aligned}
$$

This equation describes the evolution of the oscillator state (via the amplitude quadrature) conditioned on both observed and unobserved processes.

\section{Stochastic equation for the joint qubit-oscillator state}

The joint system (supersystem) evolution conditioned by all processes is found by joining the stochastic master equation [Eq. (37)] and the combined classical evolution [Eq. (B9)]. This step is performed via ${ }^{21,23}$

$$
\begin{aligned}
\bar{\rho}_{\mathcal{V}, \mathrm{c}}(\tilde{x})+\mathrm{d} \bar{\rho}_{\mathcal{V}, \mathrm{c}}(\tilde{x})= & {\left[\bar{P}_{\mathcal{V}, \mathrm{c}}(\tilde{x})+\mathrm{d} \bar{P}_{\mathcal{V}, \mathrm{c}}(\tilde{x})\right] } \\
& \times\left[\bar{\rho}_{\mathrm{c}}(t)+\mathrm{d} \bar{\rho}_{\mathrm{c}}(t)\right] .
\end{aligned}
$$

The coupled noise process in $\mathrm{d} \bar{P}_{\mathcal{V}, \mathrm{c}}$ [Eq. (B9)] and $\mathrm{d} \bar{\rho}_{\mathrm{c}}$ [Eq. (34)] is $J^{\sin }(t)$. This is the term from which correlations between the circuit and qubit evolution arise in our joint stochastic equation. This equation describes the evolution of the joint quantum-classical state conditioned on the observed process $\mathcal{V}(t)$ and the unobserved process $J^{\sin }(t)$. To remove the conditioning on the unobserved process, we simply average over it. In our approach, the unobserved process is ostensibly a white noise of zero 
mean (since we use the linear equation). The result is the following superoperator Zakai equation

$$
\begin{aligned}
\mathrm{d} \bar{\rho}_{\mathcal{V}}(\tilde{x})=\mathrm{d} & \left.t-\frac{\partial}{\partial \tilde{x}} m_{\tilde{x}}+\frac{B_{\sin }^{2}}{2} \frac{\partial^{2}}{\partial \tilde{x}^{2}}+\mathcal{V} \lambda_{\tilde{x}}+\tilde{\mathcal{L}}\right\} \bar{\rho}_{\mathcal{V}}(\tilde{x}) \\
& -\mathrm{d} t \sqrt{\eta \kappa} B_{\sin } \frac{\partial}{\partial \tilde{x}}\left[\hat{\sigma}_{z} \bar{\rho}_{\mathcal{V}}(\tilde{x})+\bar{\rho}_{\mathcal{V}}(\tilde{x}) \hat{\sigma}_{z}\right] / 2,
\end{aligned}
$$

where $\eta$ is defined in Eq. (35), and $\tilde{\mathcal{L}}$ is defined in Eq. (36).

This description of the joint quantum-classical system evolution conditioned on the observed output $\mathcal{V}(t)$ does not preserve normalization of the state. This is because ostensible statistics were chosen for $\mathcal{V}(t)$. To complete the realistic quantum trajectory derivation, we must next find the true statistics for $\mathcal{V}(t)$, and normalize the superoperator Zakai equation.

\section{Superoperator Kushner-Stratonovich equation}

Normalization of the superoperator Zakai equation is performed $^{21}$ by taking the trace over the qubit and integrating over the oscillator $(\tilde{x})$ :

$$
\rho_{\mathcal{V}}(\tilde{x})+\mathrm{d} \rho_{\mathcal{V}}(\tilde{x})=\frac{\bar{\rho}_{\mathcal{V}}(\tilde{x})+\mathrm{d} \bar{\rho}_{\mathcal{V}}(\tilde{x})}{\int \operatorname{Tr}\left[\bar{\rho}_{\mathcal{V}}(\tilde{x})+\mathrm{d} \bar{\rho}_{\mathcal{V}}(\tilde{x})\right] d \tilde{x}}
$$

It is important to note that the resulting equation is not the superoperator Kushner-Stratonovich (SKS) equation because ostensible statistics are still being used for the realistic homodyne output $\mathcal{V}(t)$. To find the SKS equation, we must substitute the true expression for $\mathcal{V} \mathrm{d} t$. The true distribution for $\mathcal{V}$ is found from the superoperator Zakai equation (B11) by tracing over the qubit and integrating over all $\tilde{x}$, then multiplying the result by the ostensible distribution $\Lambda(\mathcal{V})$ [Eq. (B5)]. That is,

$$
\begin{aligned}
P(\mathcal{V}) & =\Lambda(\mathcal{V}) \int \operatorname{Tr}\left[\bar{\rho}_{\mathcal{V}}(\tilde{x})+\mathrm{d} \bar{\rho}_{\mathcal{V}}(\tilde{x})\right] d \tilde{x} \\
& =\frac{\sqrt{\mathrm{d} t}}{\sqrt{2 \pi}} \exp \left\{-\left[\mathcal{V}-\lambda_{\langle\tilde{x}\rangle_{\rho}}\right]^{2} \mathrm{~d} t / 2\right\}
\end{aligned}
$$

where $\lambda_{\langle\tilde{x}\rangle_{\rho}} \equiv \sqrt{A_{\mathrm{SN}}} \gamma\langle\tilde{x}\rangle_{\rho} / 2 B_{\text {sin }}$ [c.f. Eq. (41)], and the average $\langle\tilde{x}\rangle_{\rho}=\int \tilde{x} \operatorname{Tr}[\rho(\tilde{x})] \mathrm{d} \tilde{x}$ is qubit-dependent (hence the $\rho$ subscript). This means that the true expression for $\mathcal{V}(t)$ is

$$
\mathcal{V}(t) \mathrm{d} t=\lambda_{\langle\tilde{x}\rangle_{\rho}} \mathrm{d} t+\mathrm{d} \mathcal{W}(t)
$$

where $\mathrm{d} \mathcal{W}(t)$ is the white noise Wiener increment a realistic observer would see. The end result is the SKS equation (42).
* Electronic address: n.oxtoby@herts.ac.uk

† Electronic address: h.wiseman@griffith.edu.au

1 B. E. Kane, Nature (London) 393, 133 (1998).

2 D. Loss and D. P. DiVincenzo, Phys. Rev. A 57, 120 (1998).

3 V. Privman, I. D. Vagner, and G. Kventsel, Phys. Lett. A 239, 141 (1998).

4 A. Imamoḡlu, D. D. Awschalom, G. Burkard, D. P. DiVincenzo, D. Loss, M. Sherwin, and A. Small, Phys. Rev. Lett. 83, 4204 (1999).

${ }^{5}$ R. Vrijen, E. Yablonovitch, K. Wang, H. W. Jiang, A. Balandin, V. Roychowdhury, T. Mor, and D. DiVincenzo, Phys. Rev. A 62, 012306 (2000).

${ }^{6}$ H. J. Carmichael, An Open Systems Approach to Quantum (Springer, Berlin, 1993).

7 H. M. Wiseman and G. J. Milburn, Phys. Rev. A 47, 1652 (1993).

8 H. M. Wiseman, Quantum Semiclass. Opt. 8, 205 (1996).

9 A. N. Korotkov, Phys. Rev. B 60, 5737 (1999).

10 A. N. Korotkov, Phys. Rev. B 63, 115403 (2001).

11 H. M. Wiseman, D. Wahyu Utami, H. B. Sun, G. J. Milburn, B. E. Kane, A. Dzurak, and R. G. Clark, Phys. Rev. B 63, 235308 (2001).

12 H.-S. Goan, G. J. Milburn, H. M. Wiseman, and H. B. Sun, Phys. Rev. B 63, 125326 (2001).

13 A. N. Korotkov, Phys. Rev. B 64, 193407 (2001).

14 H.-S. Goan and G. J. Milburn, Phys. Rev. B 64, 235307 (2001).

15 A. N. Korotkov, Phys. Rev. B 67, 235408 (2003).
16 A. N. Korotkov, in Quantum Noise in Mesoscopic Physics, edited by Y. V. Nazarov (Kluwer Academic, Netherlands, 2003), pp. 205-228.

17 T. M. Stace and S. D. Barrett (2004), cond-mat/0309610.

18 T. M. Stace and S. D. Barrett, Phys. Rev. Lett. 92, 136802 (2004).

19 H.-S. Goan, Phys. Rev. B 70, 075305 (2004).

20 J. Gambetta, A. Blais, M. Boissonneault, A. A. Houck, D. I. Schuster, and S. M. Girvin, Physical Review A 77, 012112 (2008).

21 N. P. Oxtoby, P. Warszawski, H. M. Wiseman, H.-B. Sun, and R. E. S. Polkinghorne, Phys. Rev. B 71, 165317 (2005).

22 P. Warszawski, H. M. Wiseman, and H. Mabuchi, Phys.

opticRev. A 65, 023802 (2002).

${ }^{23}$ P. Warszawski and H. M. Wiseman, J. Opt. B: Quantum Semiclass. Opt. 5, 1 (2003).

24 G. Zimmerli, T. M. Eiles, R. L. Kautz, and J. M. Martinis, Appl. Phys. Lett. 61, 237 (1992).

25 H. Wong, Microelectronics Reliability 43, 585 (2003).

26 R. J. Schoelkopf, P. Wahlgren, A. A. Kozhevnikov, P. Delsing, and D. E. Prober, Science 280, 1238 (1998).

27 A. Aassime, G. Johansson, G. Wendin, R. J. Schoelkopf, and P. Delsing, Phys. Rev. Lett. 86, 3376 (2001).

28 H. Qin and D. A. Williams, Appl. Phys. Lett. 88, 203506 (2006).

29 J. Gambetta and H. M. Wiseman, J. Opt. B: Quantum Semiclass. Opt. 7, S250 (2005).

30 C. Ahn, H. M. Wiseman, and G. J. Milburn, Phys. Rev. A 67, 052310 (2003). 
31 M. Sarovar, C. Ahn, K. Jacobs, and G. J. Milburn, Phys. Rev. A 69, 052324 (2004).

32 C. Ahn, H. M. Wiseman, and K. Jacobs, Phys. Rev. A 70, 024302 (2004).

33 R. van Handel and H. Mabuchi (2005), quant-ph/0511221.

${ }^{34}$ H. M. Wiseman and G. J. Milburn, Phys. Rev. Lett. 70, 548 (1993).

35 H. M. Wiseman, Phys. Rev. Lett. 75, 4587 (1995).

36 A. C. Doherty and K. Jacobs, Phys. Rev. A 60, 2700 (1999).

37 A. C. Doherty, S. Habib, K. Jacobs, H. Mabuchi, and S. M. Tan, Phys. Rev. A 62, 012105 (2000).

38 M. A. Armen, J. K. Au, J. K. Stockton, A. C. Doherty, and H. Mabuchi, Phys. Rev. Lett. 89, 133602 (2002).

39 H. M. Wiseman, S. Mancini, and J. Wang, Phys. Rev. A 66, 013807 (2002).

40 W. P. Smith, J. E. Reiner, L. A. Orozco, S. Kuhr, and H. M. Wiseman, Phys. Rev. Lett. 89, 133601 (2002).

41 R. Ruskov and A. N. Korotkov, Phys. Rev. B 66, 041401(R) (2002).

42 K. Jacobs, Phys. Rev. A 67, 030301(R) (2003).

43 J. Combes and K. Jacobs, Phys. Rev. Lett. 96, 010504 (2006).

44 A. Aassime, D. Gunnarsson, K. Bladh, P. Delsing, and R. J. Schoelkopf, Appl. Phys. Lett. 79, 4031 (2001).

${ }^{45}$ T. Hayashi, T. Fujisawa, H. D. Cheong, Y. H. Jeong, and Y. Hirayama, Phys. Rev. Lett. 91, 226804 (2003).

46 J. Gorman, D. G. Hasko, and D. A. Williams, Phys. Rev. Lett. 95, 090502 (2005).

47 D. J. Reilly, C. M. Marcus, M. P. Hanson, and A. C. Gossard, Appl. Phys. Lett. 91, 162101 (2007).

48 V. O. Turin and A. N. Korotkov, Phys. Rev. B 69, 195310
(2004)

49 K. Bladh, T. Duty, D. Gunnarsson, and P. Delsing, New Journal of Physics 7, 180 (2005).

${ }^{50}$ H. M. Wiseman and G. J. Milburn, Phys. Rev. A 47, 642 (1993).

51 D. M. Pozar, Microwave Engineering (Wiley, New York, 1998).

52 D. V. Averin and E. V. Sukhorukov, Phys. Rev. Lett. 95, 126803 (2005).

53 N. P. Oxtoby, H. M. Wiseman, and H.-B. Sun, Phys. Rev. B 74, 045328 (2006).

${ }^{54}$ C. W. Gardiner and P. Zoller, Quantum Noise (Springer, Berlin, 2000), 2nd ed.

55 D. Gatarek and N. Gisin, Journal of Mathematical Physics 32, 2152 (1991).

56 N. P. Oxtoby, Ph.D. thesis, Griffith University (2006).

57 P. Goetsch and R. Graham, Phys. Rev. A 50, 5242 (1994).

58 J. Gambetta and H. M. Wiseman, Phys. Rev. A 64, 042105 (2001).

59 M. Büttiker, Phys. Rev. B 46, 12485 (1992).

60 T. Fujisawa and Y. Hirayama, Appl. Phys. Lett. 77, 543 (2000).

61 H. D. Cheong, T. Fujisawa, T. Hayashi, Y. Hirayama, and Y. H. Jeong, Appl. Phys. Lett. 81, 3257 (2002).

62 The choice $e>0$ corresponds to defining current in terms of the direction of electron flow. That is, in the opposite direction to conventional current.

63 The work of Büttiker in Ref. 59, for example, is a more general reference for QPC conductance calculated using scattering theory.

64 There is also a technique involving the transmission of $\mathrm{rf}$ radiation. See Refs. 28, 60, and 61, for example. 